\title{
Growth mode transition from layer by layer to step flow during the growth of heteroepitaxial $\mathrm{SrRuO}_{3}$ on (001) $\mathrm{SrTiO}_{3}$
}

\author{
J. Choi and C. B. Eom ${ }^{\text {a) }}$ \\ Department of Materials Science and Engineering, University of Wisconsin-Madison, Madison, \\ Wisconsin 53706 \\ G. Rijnders, H. Rogalla, and D. H. A. Blank \\ Low Temperature Division and MESA Research Institute, University of Twente, P.O. Box 217, 7500 AE \\ Enschede, The Netherlands
}

(Received 1 September 2000; accepted for publication 4 June 2001)

\begin{abstract}
We have observed the growth mode transition from two-dimensional (2D) layer-by-layer to step-flow in the earliest stage growth of heteroepitaxial $\mathrm{SrRuO}_{3}$ thin films on $\mathrm{TiO}_{2}$-terminated (001) $\mathrm{SrTiO}_{3}$ substrates by in situ high pressure reflective high energy electron diffraction (RHEED) and atomic-force microscopy. There is no RHEED intensity recovery after each laser pulse in the first oscillation when the growth mode is 2D layer-by-layer. On the other hand, it is getting more pronounced in the second oscillation, and finally reaches a dynamic steady state in which the growth mode is completely changed into the step-flow mode. The origin of the growth mode transition can be attributed to a change in the mobility of adatoms and switching the surface termination layer from the substrate to the film. $\mathrm{SrRuO}_{3}$ thin films with an atomically smooth surface grown by atomic layer control can be used in oxide multilayered heterostructure devices. (C) 2001 American Institute of Physics. [DOI: 10.1063/1.1389837]
\end{abstract}

Epitaxial multilayered heterostructures based on perovskite oxides have a great potential for future electronic device applications such as spin-polarized tunnel junction. ${ }^{1-3}$ In such a tunnel junction, the junction magnetoresistance (JMR) is determined by the difference in the density of states for tunneling. Highly spin-polarized magnetic oxides, halfmetallic oxides, are expected to exhibit large, almost infinite JMR in the Julliere model. ${ }^{4}$ In reality, it has been shown that there is a strong temperature dependence of the JMR in the doped manganite system, i.e., premature loss below the $\mathrm{Cu}$ rie temperature. ${ }^{1}$ This result is important for technological applications and depends on the quality of the junctions: uniformity and quality of barrier layer, interface sharpness, and surface magnetization. For the study of its physical origin, the sharp interface and defect-free barrier layer in the trilayered tunnel junction are required and must be controlled on an atomic scale.

Significant progress has been made in studying growth mechanisms of homoepitaxial oxide thin films such as $\mathrm{SrTiO}_{3}$, using molecular-beam epitaxy (MBE), ${ }^{5}$ laser $\mathrm{MBE},{ }^{6,7}$ and pulsed-laser deposition (PLD). ${ }^{8}$ In all papers, the surface mobility of adatoms during deposition plays an important role in determining the growth modes. In this study, we will focus on the heteroepitaxy of $\mathrm{SrRuO}_{3}$ films grown on single-terminated $\mathrm{SrTiO}_{3}$ substrates and the applicability of the oxide multilayered heterostructures.

Among many perovskite oxides, the itinerant metallic ferromagnetic oxide $\mathrm{SrRuO}_{3}$ is an ideal system to study heteroepitaxial thin film growth. Not only is it chemically stable, making ex situ scanning probe microscopy (SPM) measurements possible, it is also structurally similar to the

${ }^{a)}$ Electronic mail: eom@engr.wisc.edu
$\mathrm{SrTiO}_{3}$ substrate with a lattice mismatch of only $0.64 \%$. Moreover, epitaxial single-crystal $\mathrm{SrRuO}_{3}$ thin films can be coherently grown on miscut $\mathrm{SrTiO}_{3}$ substrates. ${ }^{9,10}$ In this study, we have investigated the initial stage nucleation and growth of the heteroepitaxial $\mathrm{SrRuO}_{3}$ thin films by in situ high pressure reflective high energy electron diffraction (RHEED) and atomic force microscopy (AFM). ${ }^{11}$

Epitaxial $\mathrm{SrRuO}_{3}$ thin films were grown on $\mathrm{TiO}_{2}$-terminated (001) $\mathrm{SrTiO}_{3}$ substrates by PLD using a $\mathrm{KrF}$ excimer laser $(\lambda=248 \mathrm{~nm})$ with an energy density of 2.5

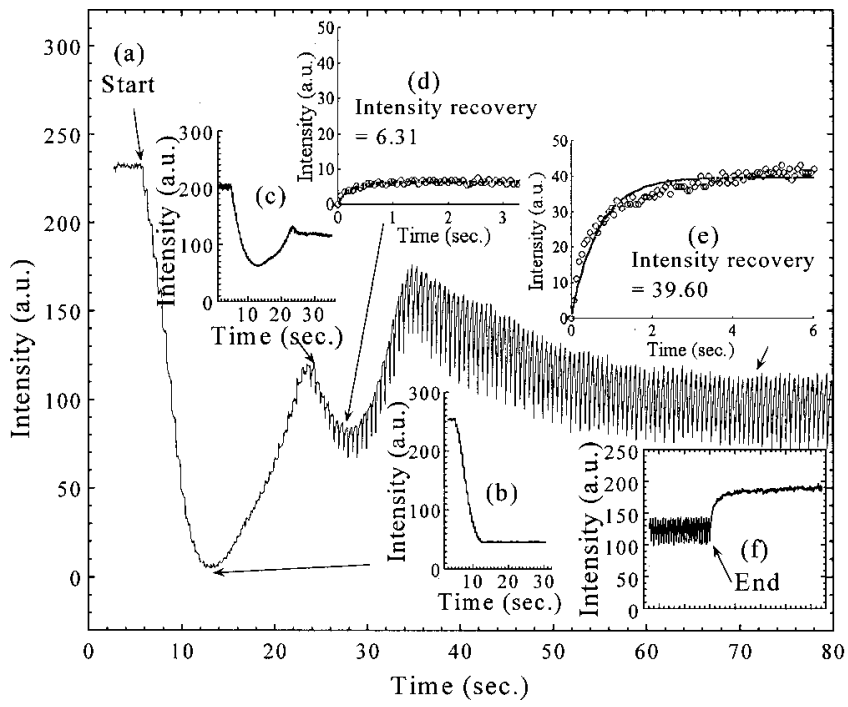

FIG. 1. Overall RHEED intensity variations during the heteroepitaxial growth of the $\mathrm{SrRuO}_{3}$ film on the $\mathrm{TiO}_{2}$-terminated (001) $\mathrm{SrTiO}_{3}$ substrate. Insets show the RHEED intensity recovery after the growth is interrupted at the bottom region of the first oscillation (b), at the top region of the first oscillation (c), at the bottom region of the second oscillation (d), in the steady state region (e), and finally stopped (f). 

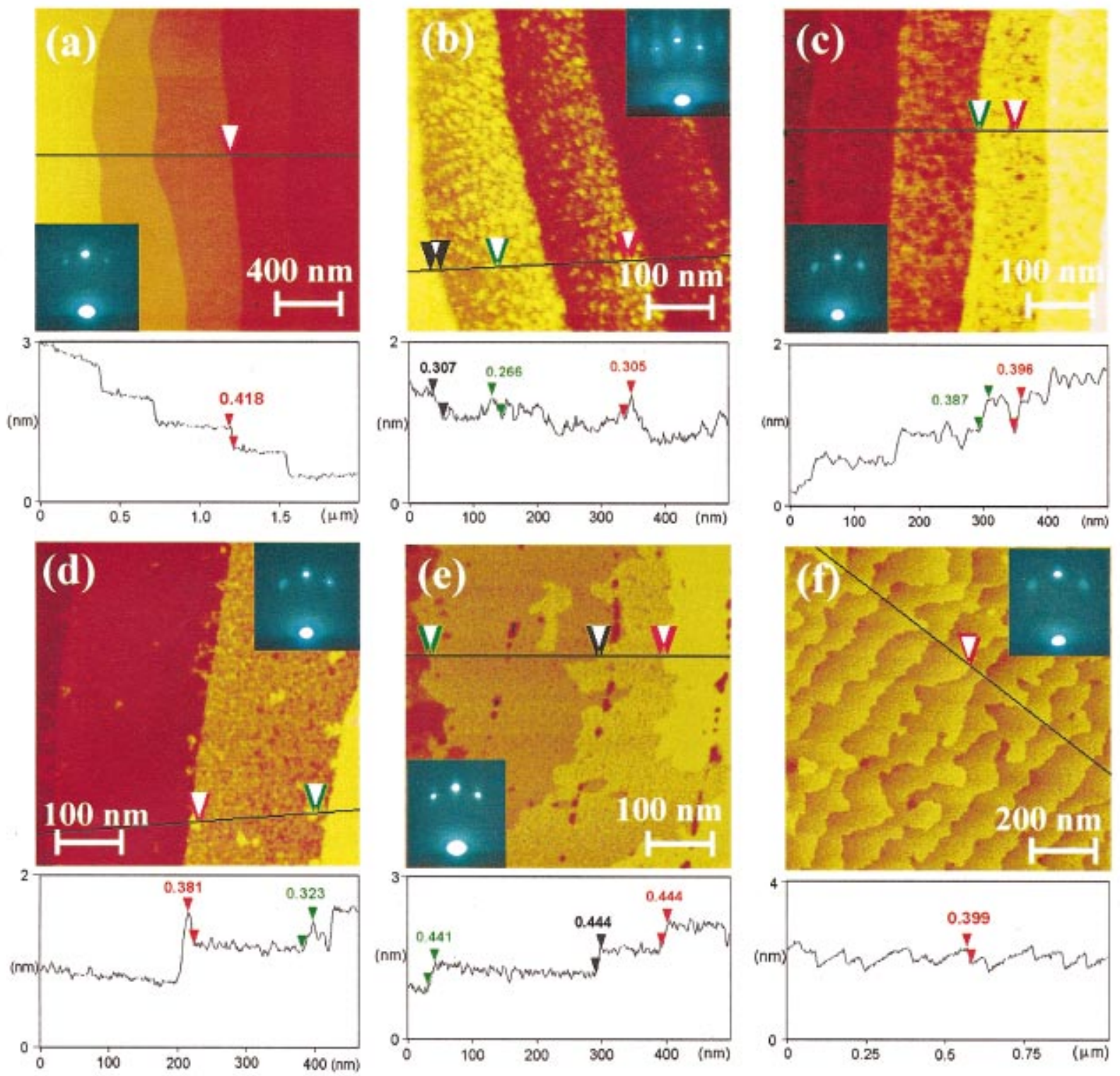

FIG. 2. (Color) AFM images and RHEED patterns of the as-grown films obtained after interrupting the growth at each stage, as marked in Fig. 1.

$\mathrm{J} / \mathrm{cm}^{2}$ at a repetition rate of $2 \mathrm{~Hz}$. The single $\mathrm{TiO}_{2}$-terminated surface of the (001) $\mathrm{SrTiO}_{3}$ substrates has been obtained in a three-step treatment which was developed by Koster et al.: ${ }^{12}$ soak in water, chemical etch with a $\mathrm{NH}_{4} \mathrm{~F}$ buffered $\mathrm{HF}$ solution, and postanneal at $950^{\circ} \mathrm{C}$ for $1 \mathrm{~h}$ under an oxygen atmosphere. The miscut angle of the substrate ranges from $0.05^{\circ}$ to $0.2^{\circ}$. The growth temperature and oxygen pressure were kept at $600{ }^{\circ} \mathrm{C}$ and 100 mTorr, respectively.

Figure 1 shows the overall RHEED intensity variations during the growth of the $\mathrm{SrRuO}_{3}$ film on the $\mathrm{TiO}_{2}$-terminated (001) $\mathrm{SrTiO}_{3}$ substrate with a miscut angle of approximately $0.2^{\circ}$. The insets show the RHEED intensity recovery after interrupting the growth. The overall RHEED intensity oscillated twice and then reached a steady state until the growth was stopped. This transient behavior of the overall RHEED intensity represents the fact that the growth mode of the $\mathrm{SrRuO}_{3}$ film has a transition from the two-dimensional (2D) layer-by-layer to the step-flow mode. In addition to such a change in the overall RHEED intensity, the different mobility behaviors of adatoms can be seen from the intensity reDownloaded 02 Mar 2007 to 128.104.198.190. Redistribution subject coveries obtained after interrupting the growth. There is almost no intensity recovery at the bottom and top regions of the first oscillation [Figs. 1(b) and 1(c)]. Instead, the intensity recoveries after interrupting the growth are getting more pronounced from the region of the second oscillation, and finally reaches a steady state [Figs. 1(d), 1(e), and 1(f)]. These results indicate a change of the mobility of the adatoms, which can lead to a change in the growth mode.

In order to verify the growth mode transition in this earliest stage, the surface morphologies of the as-grown films were studied by tapping mode AFM. Figure 2 shows microstructure evolutions and RHEED patterns obtained from each stage of the growth, as marked in Fig. 1. Figure 2(a) shows the perfect $\mathrm{TiO}_{2}$-terminated surface of the (001) $\mathrm{SrTiO}_{3}$ substrate. One can see clear step-terrace structures with only one unit-cell height. The RHEED pattern shows sharp spots lying on the zeroth Laue circle, indicating that the $\mathrm{SrTiO}_{3}$ surface is atomically smooth. Figure 2(b) shows the surface morphology at the bottom region of the first oscillation [position as indicated by Fig. 1(b)]. The AFM image shows many $2 \mathrm{D}$
to AIP license or copyright, see http://apl.aip.org/apl/copyright.jsp 
islands randomly nucleated and distributed on the $\mathrm{SrTiO}_{3}$ substrate terraces. The RHEED pattern has additional diffuse spots, originating from the transmission of the electron beam through the small 2D islands. Figure 2(c) shows the surface morphology at the maximum of the first RHEED intensity oscillation, indicated by Fig. 1(c). The $\mathrm{SrRuO}_{3}$ film nearly covers all of substrate terraces, indicating the 2D layer-bylayer growth mode at this initial growth. The RHEED intensity oscillation is in agreement with the observed surface structure changes.

The AFM image [Fig. 2(d)] taken at the minimum of the second oscillation shows a remarkable difference compared to Fig. 2(b). It can be seen that the $\mathrm{SrRuO}_{3}$ film is completely covered. In addition, more 2D islands start to nucleate at the step ledge regions where there are more favorable sites for nucleation. However, some 2D islands are still visible on the terraces, causing the oscillating behavior of the RHEED intensity. Note that the number of islands is much less or lower compared to the surface morphology as obtained at the minimum of the first oscillation. Figure 2(e) taken from the steady state region of the RHEED intensity indicated by Fig. 1(e) shows that the $\mathrm{SrRuO}_{3}$ film grows by the step-flow mode. The original step structure of the $\mathrm{SrTiO}_{3}$ substrate can be seen as holes at the ledges. Finally, the surface morphology and RHEED pattern of a $56 \mathrm{~nm}$ thick $\mathrm{SrRuO}_{3}$ film [Fig. 2(f)] are almost identical to those of the treated (001) $\mathrm{SrTiO}_{3}$ substrate. Due to the step-flow growth, the atomically smooth surface structure and single domain structure of the $\mathrm{SrRuO}_{3}$ film can be obtained. Only one unitcell high steps without step bunching imply the single terminated surface of the $\mathrm{SrRuO}_{3}$ film. From the RHEED intensity variations and the microstructure evolutions, it is clear that the growth mode of heteroepitaxial $\mathrm{SrRuO}_{3}$ thin films makes a change from the 2D layer-by-layer to the step-flow, and that there is a close relationship between the growth mode transition and the change in the mobility of the adatoms.

To elucidate this relationship, we must consider experimental factors that govern the growth mode of thin films on vicinal substrates. The growth mode of the thin films is principally determined by growth temperature, lattice mismatch, substrate miscut angle, growth rate, and surface chemistry. In homoepitaxy, Lippmaa et al. already reported the effects of the growth temperature and repetition rate on the growth mode change in the homoepitaxial $\mathrm{SrTiO}_{3}$ thin films. ${ }^{8}$ At repetition rates of $5 \mathrm{~Hz}$ or less the growth mode changed gradually from 2D layer-by-layer to step-flow as the growth temperature was increased. Especially, in the step-flow mode regime there is no growth mode transition from the 2D layerby-layer mode to the step-flow at the earliest stage growth. In their case, the change in the growth mode is only dependent on the growth conditions, like temperature and growth rate. This behavior is completely different from what we observe during the heteroepitaxial growth of $\mathrm{SrRuO}_{3}$ on (001) $\mathrm{SrTiO}_{3}$ : the growth mode transition from $2 D$ layer-by-layer to step flow in the earliest stage growth.

In the case of heteroepitaxy of layered perovskite oxides, the termination layer of the substrate can dominantly influence the growth kinetics at the earliest stage such as wettability, mobility, and sticking coefficient. From the AFM im- ages in Fig. 2 and RHEED intensity recovery data, we conclude that the mobility of the adatoms deposited on the $\mathrm{TiO}_{2}$-terminated $\mathrm{SrTiO}_{3}$ substrates is significantly lower compared to that of the steady state regime. The mobility of the adatoms deposited on the $\mathrm{SrRuO}_{3}$ film is greatly enhanced and reaches the steady state as the $\mathrm{TiO}_{2}$ termination layer of the $\mathrm{SrTiO}_{3}$ substrate is covered by the $\mathrm{SrRuO}_{3}$ film. In addition to the growth mode transition at the earliest stage, the data of Fig. 1 shows another feature. The number of laser pulses to complete the first RHEED oscillation (34 pulses) differs significantly from that to complete the second RHEED oscillation (22 pulses). Also, this number is not consistent with the number of 22 pulses per unit cell, as calculated from low angle x-ray diffraction measurements. Such a discrepancy could be associated with desorption of Ru adatoms. We will discuss the details of this result elsewhere. ${ }^{13}$

In conclusion, we report the observation of the growth mode transition from the 2D layer-by-layer mode to the stepflow during the earliest stage growth of the heteroepitaxial $\mathrm{SrRuO}_{3}$ films on the $\mathrm{TiO}_{2}$-terminated (001) $\mathrm{SrTiO}_{3}$ substrate. Such a transition during the earliest stage is a unique phenomenon in heteroepitaxy. This work shows that the growth kinetics at the earliest stage growth of heteroepitaxy can have a large influence on the microstructures and interfaces in multilayered heterostructures. For example, $\mathrm{SrRuO}_{3}$ thin films grown on $\mathrm{TiO}_{2}$-terminated (001) $\mathrm{SrTiO}_{3}$ substrates by atomic layer control have an atomically smooth surface and single domain structure. $\mathrm{SrRuO}_{3}$ films with a smooth surface can be used as electrode layers in spin-polarized tunnel junctions requiring a sharp interface and uniform coverage of barrier layers.

The authors would like to thank Frank Roesthuis and Gertjan Koster for helpful discussions. This research was supported by NSF Grant Nos. DMR 9802444 and 9902444 , the David and Lucile Packard Fellowship (C.B.E.), and the NFS Young Investigator Award (C.B.E.).

${ }^{1}$ J. Z. Sun, W. J. Gallagher, P. R. Duncombe, L. Krusin-Elbaum, R. A. Altman, A. Gupta, Yu Lu, G. Q. Gong, and Gang Xiao, Appl. Phys. Lett. 69, 3266 (1996).

${ }^{2}$ J. O'Donnell, A. E. Andrus, S. Oh, E. V. Colla, and J. N. Eckstein, Appl. Phys. Lett. 76, 1914 (2000).

${ }^{3}$ T. Obata, T. Manako, Y. Shimakawa, and Y. Kubo, Appl. Phys. Lett. 74, 290 (1999).

${ }^{4}$ M. Julliere, Phys. Lett. 61, 2472 (1975).

${ }^{5}$ M. Naito, H. Yamamoto, and H. Sato, Physica C 305, 233 (1998).

${ }^{6}$ M. Lippmaa, N. Nakagawa, M. Kawasaki, S. Ohashi, Y. Inaguma, M. Itoh, and H. Koinuma, Appl. Phys. Lett. 74, 3543 (1999).

${ }^{7}$ M. Lippmaa, N. Nakagawa, M. Kawasaki, S. Ohashi, and H. Koinuma, Appl. Phys. Lett. 76, 2439 (2000).

${ }^{8}$ G. Koster, G. J. H. M. Rijnders, D. H. A. Blank, and H. Rogalla, Appl. Phys. Lett. 74, 3729 (1999).

${ }^{9}$ C. B. Eom, R. J. Cava, R. M. Fleming, J. M. Phillips, R. B. van Dover, J. H. Marshall, J. W. P. Hsu, J. J. Krajewski, and W. F. Peck, Jr., Science 258, 1766 (1992).

${ }^{10}$ Q. Gan, R. A. Rao, and C. B. Eom, Appl. Phys. Lett. 70, 1962 (1997).

${ }^{11}$ A. J. H. M. Rijnders, G. Koster, D. H. A. Blank, and H. Rogalla, Appl. Phys. Lett. 70, 1888 (1997).

${ }^{12}$ G. Koster, B. L. Kropman, A. J. H. M. Rijnders, D. H. A. Blank, and H. Rogalla, Appl. Phys. Lett. 73, 2920 (1998).

${ }^{13}$ G. Rijnders, D. H. A. Blank, H. Rogalla, J. Choi, and C. B. Eom (unpublished). 